\title{
Comparison of Complications in Stroke Subjects Undergoing Early Versus Standard Tracheostomy
}

\author{
Young Chan Lee MD PhD, Tae Hyun Kim MD, Jung-woo Lee DMD MSD PhD, \\ In-Hwan Oh MD, and Young Gyu Eun MD PhD
}

\begin{abstract}
BACKGROUND: Although the benefits of early tracheostomy have been discussed in numerous studies, it is still unclear whether it is safe to perform early tracheostomy on unstable stroke patients. The purpose of this study is to assess the influences of the timing of tracheostomy on the incidence of complications following surgical tracheostomy in stroke patients. METHODS: We retrospectively performed chart reviews of 95 stroke subjects who underwent tracheostomy. In terms of timing, procedures performed within $7 \mathrm{~d}$ of intubation were categorized as early tracheostomy, and those performed after $7 \mathrm{~d}$ were categorized as standard tracheostomy. The incidence of complications following tracheostomy was compared between the two groups. The risk factors for complication were also investigated. RESULTS: Among the 95 subjects, $59(62.1 \%)$ received early tracheostomy and $36(37.9 \%)$ received standard tracheostomy. The overall incidence of tracheostomy complications was $\mathbf{2 4 . 2 \%}$, and there was no significant difference in incidence between the two groups. A comparison of risk factors between the groups with and without complications revealed no significant differences in age, sex, body mass index, Glasgow coma scale score, stroke type, or history of underlying disease. However, activated partial thromboplastin time was significantly higher in the group with complication. CONCLUSIONS: There was no significant difference in the incidence of complications in stroke subjects undergoing early versus standard tracheotomy. Key words: early tracheostomy; standard tracheostomy; tracheostomy complications. [Respir Care 2015;60(5):651-657. (C) 2015 Daedalus Enterprises]
\end{abstract}

\section{Introduction}

The prognosis for stroke patients who require mechanical ventilation is poor, and proper airway management is crucial for providing better clinical outcomes. Of these airway management methods, tracheostomy is commonly performed on patients who require prolonged mechanical ventilation. Tracheostomy reduces total mechanical ventilation time, shortens ICU and hospital stays, and is known to effectively reduce the occurrence of both pneumonia and hospital mortality. ${ }^{1-6}$

A recent study examined the benefits of early tracheostomy, reporting that tracheostomy performed on stroke

Drs YC Lee, Kim, and Eun are affiliated with the Department of Otolaryngology-Head and Neck Surgery, School of Medicine, Kyung Hee University; Dr J Lee is affiliated with the Department of Oral and Maxillofacial Surgery, School of Dentistry, Kyung Hee University; Dr Oh is affiliated with the Department of Preventive Medicine, School of Medicine, Kyung Hee University, Seoul, Republic of Korea.

Drs YC Lee and Kim are co-first authors of this paper. subjects within $1-3 \mathrm{~d}$ of intubation reduced ICU mortality, use of sedatives, and 6-month mortality. ${ }^{7}$ In addition to these effects, another study reported that early tracheostomy in subjects who required postoperative mechanical ventilation following cardiac surgery effectively reduced hospital mortality, cardiac mortality, and the duration of ICU and hospital stays as compared with subjects who received late tracheostomy. ${ }^{8}$

Despite the evident benefits of tracheostomy, the complications that can arise as a result of this procedure cannot be ignored. Tracheostomy complications may be categorized as early or late complications. Early complications

\footnotetext{
The authors have disclosed no conflicts of interest.

Correspondence: Young Gyu Eun MD PhD, Department of Otolaryngology-Head and Neck Surgery, Kyung Hee University School of Medicine, No. 1 Hoegi-dong, Dongdaemun-gu, Seoul 130-702, Republic of Korea. E-mail: ygeun@hanmail.net.
}

DOI: $10.4187 /$ respcare. 03652 
include hemorrhage, wound infection, subcutaneous emphysema, tube obstruction, and early tube displacement, whereas late complications include difficulty swallowing, tracheal stenosis, tracheoinnominate artery fistula, tracheoesophageal fistula, granuloma formation, and persistent stoma. ${ }^{9}$

Although the benefits of early tracheostomy have been discussed in numerous studies and its advantages have been confirmed, it remains uncertain whether it is safe to perform early tracheostomy on unstable stroke patients. Therefore, we assessed the effect of the timing of tracheostomy on the incidence of complications following surgical tracheostomy in stroke subjects.

\section{Methods}

This study was conducted through a retrospective chart review of stroke patients who underwent tracheostomy at the Kyung Hee University Medical Center (Seoul, Republic of Korea) between January 2011 and December 2012.

\section{Subjects}

This study included 95 consecutive stroke subjects who were diagnosed with intracerebral hemorrhage, subarachnoid hemorrhage, or ischemic stroke. All of these subjects subsequently required mechanical ventilation and underwent a surgical tracheostomy during the study period. Patients who required immediate tracheostomy or had existing respiratory disease were excluded from the study. Subject age, sex, body mass index (BMI), Glasgow coma scale score, history of underlying disease (hypertension, diabetes mellitus, and heart disease), prothrombin time, activated partial thromboplastin time (aPTT), international normalized ratio, and platelet count were investigated, and correlations between these factors and the incidence of tracheostomy complications were evaluated. Moreover, duration of intubation, follow-up after tracheostomy, and decannulation status were also investigated. The institutional review boards of Kyung Hee University Hospital approved the protocol.

\section{Tracheostomy Timing and Procedure}

Regarding the timing of tracheostomy, cases in which tracheostomy was performed within $7 \mathrm{~d}$ of intubation were classified as early tracheostomy, and cases in which tracheostomy was performed after $7 \mathrm{~d}$ since intubation were classified as standard tracheostomy. All tracheostomies were performed by experienced otolaryngologists or neurosurgeons after informed consent had been obtained from the subject's guardian. Additionally, the procedure itself did not differ greatly between the subjects. All surgical tracheostomies were performed in the ICU according

\section{QUICK LOOK}

\section{Current knowledge}

The timing of tracheostomy in mechanically ventilated patients remains controversial. Patient characteristics are likely as important as timing. In particular, patients with neurologic injury may benefit from early tracheostomy when compared with patients with primary lung failure.

\section{What this paper contributes to our knowledge}

In a retrospective analysis of subjects following stroke, there was no difference in the incidence of complications between subjects who received a tracheostomy in the first $7 \mathrm{~d}$ compared with those receiving a tracheostomy after day 7 .

to the technique previously described by Heffner et al. ${ }^{10}$ With the subject's neck fully extended, the surgeons made a 3-4-cm horizontal skin incision over the second or third tracheal rings. After division of the subcutaneous tissue, separation of the strap muscles, and ligation of the thyroid isthmus, incision of the trachea was performed at the second and third tracheal rings. With the trachea open, the endotracheal tube was pulled back so the tip was just above the tracheal opening and the tracheostomy tube was inserted. Chest x-rays were obtained immediately after the procedure and the following day.

\section{Tracheostomy Complications}

Complications following tracheostomy were categorized as early or late, depending on the timing of the occurrence. Early complications were those that occurred during the procedure or immediately after tracheostomy, and included hemorrhage, subcutaneous emphysema, tube obstruction, and tracheal wall injury. Late complications were those that occurred while the tube was in place following tracheostomy or during the long-term observation period after decannulation, and included persistent stoma, granuloma formation, tube displacement, wound infection, and tracheal stenosis. ${ }^{9}$ Persistent stoma was considered in cases of continuation of the tract after decannulation and a sufficient duration of observation period, with an ingrowth of the squamous epithelium to the trachea. Diagnosis of granuloma formation was performed by flexible bronchoscopic evaluation at the time of tube replacement or decannulation. We defined significant granuloma formation as cases where the granulation tissue obstructed the airway at the stoma and either caused difficulty replacing the tracheostomy tube or led to a delay in decannulation. 


\section{Statistical Analysis}

Statistical analyses were performed using SPSS 18.0 (SPSS, Chicago, Illinois). Categorical data were analyzed using the chi-square test, and continuous data using the independent $t$ test. In the case of multiple comparisons, Bonferroni corrections were used to reduce the risk of $\alpha$ error. The survival time in each group was compared using Kaplan-Meier curves and log-rank test. Results were presented as mean \pm SD. $P$ values $<.05$ were considered to be statistically significant.

\section{Results}

Among the 95 subjects who underwent tracheostomy, $59(62.1 \%)$ received early tracheostomy, and $36(37.9 \%)$ received standard tracheostomy. The distribution of subjects according to tracheostomy timing is shown in Figure 1. The average duration of intubation for all subjects was $7.8 \pm 7.7 \mathrm{~d}$, and the average follow-up period was $63.4 \pm 61.8 \mathrm{~d}$. During the follow-up period, 29 (30.5\%) subjects underwent decannulation, and 18 (18.9\%) subjects died.

A comparison of subject characteristics according to early versus standard tracheostomy revealed no statistically significant differences in age, sex, BMI, Glasgow coma scale score, stroke type, history of underlying disease (hypertension, diabetes mellitus, and heart disease), or perioperative use of anticoagulants (Table 1).

Among the 95 subjects who underwent tracheostomy, 23 developed complications, with an overall incidence of $24.2 \%$. Complications developed in 18 of 59 subjects who received early tracheostomy (incidence rate, 30.5\%), compared with 5 of 36 subjects who received standard tracheostomy (incidence rate, 13.9\%). The difference in the incidence of complications between the early and standard tracheostomy groups was not statistically significant (Table 2). The overall survival rate did not show a statistically significant difference between the two groups $(P=.76$; (Fig. 2).

Regarding complications that occurred in subjects undergoing early tracheostomy, persistent stoma and granuloma formation had the highest occurrence (6 subjects each, $10.2 \%$ ), followed by hemorrhage in 3 subjects (5.1\%), and wound infection, subcutaneous emphysema, and tube obstruction in one subject each $(1.7 \%)$. In the standard tracheostomy group, persistent stoma occurred in $2(5.6 \%)$ subjects, and hemorrhage, subcutaneous emphysema, and tube displacement occurred in one subject each $(2.8 \%)$ (Table 2).

We also investigated risk factors for complications. Age, sex, BMI, Glasgow coma scale score, stroke type, history of underlying disease (hypertension, heart disease, and diabetes mellitus), prothrombin time, aPTT, international

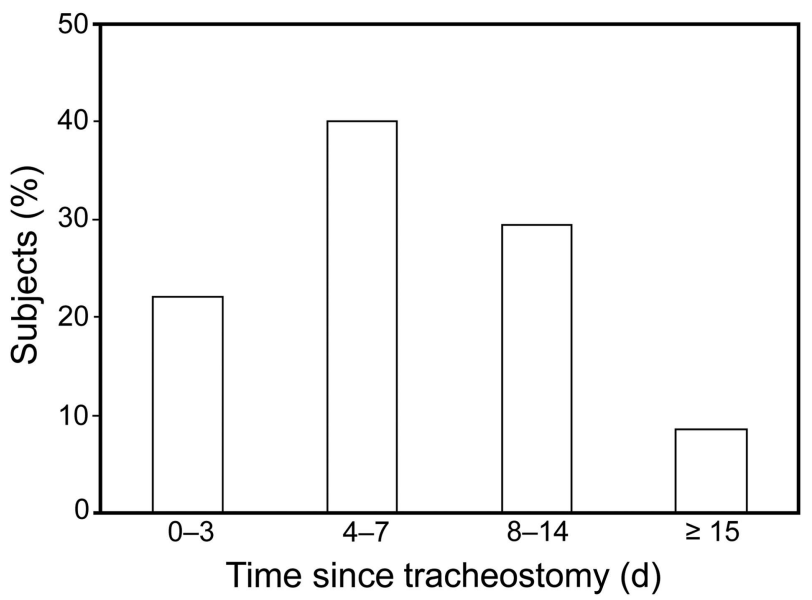

Fig. 1. Distribution of subjects according to tracheostomy timing.

normalized ratio, and platelet counts of subjects in each group were compared between subjects with and without complications and a significant difference was observed for only aPTT, which was higher in the complications group (Table 3).

\section{Discussion}

In general, replacement of an endotracheal tube with a tracheostomy may be considered for patients who require prolonged mechanical ventilation, particularly in the ICU. According to observational data, $6-11 \%$ of mechanically ventilated patients undergo tracheostomy after a median of 9-12 d. ${ }^{11-13}$ To determine the optimal timing of this procedure, studies have compared the benefits of early versus standard tracheostomy in terms of mortality, morbidity, mechanical ventilation duration, and length of ICU stay. However, data on the relationship between tracheostomy timing and adverse events are lacking. Therefore, we sought to identify and compare complications that occurred in early and standard tracheostomy groups.

Our study showed that the overall incidence rate of complications following tracheostomy was $24.2 \%$. Moreover, the incidence rates of complications in subjects undergoing early and standard tracheostomy were $30.5 \%$ and $13.9 \%$, which were not significantly different. The most frequently occurring complication was persistent stoma, which occurred in 8 out of 23 subjects who developed complications. There was a significant difference in aPTT between subjects with and without complications.

Several studies have investigated complications that arise as a result of tracheostomy. One study investigating complications that occurred within $30 \mathrm{~d}$ of the procedure reported an incidence of $4.34 \%$ (24 cases among 552 individuals). The types of complications that occurred within $30 \mathrm{~d}$ of the procedure included 9 cases of minor bleeding, 9 cases of major bleeding, 4 cases of stomal infections, 


\section{Complications in Early vs Standard Tracheostomy}

Table 1. Baseline Characteristics of Subjects

\begin{tabular}{|c|c|c|c|}
\hline & $\begin{array}{c}\text { Early } \\
\text { Tracheostomy } \\
(n=59)\end{array}$ & $\begin{array}{c}\text { Standard } \\
\text { Tracheostomy } \\
(n=36)\end{array}$ & $P$ \\
\hline Age $(y)$ & $63.69 \pm 12.27$ & $68.08 \pm 13.47$ & .10 \\
\hline Males/females & $29 / 30$ & $18 / 18$ & .93 \\
\hline BMI & $22.58 \pm 3.23$ & $23.32 \pm 3.75$ & .30 \\
\hline GCS score & $6.98 \pm 3.50$ & $7.03 \pm 4.10$ & .95 \\
\hline \multicolumn{4}{|l|}{ Stroke $(n, \%)$} \\
\hline $\mathrm{ICH}$ & $25(42.4)$ & $17(47.2)$ & .81 \\
\hline SAH & $14(23.7)$ & $9(25.0)$ & \\
\hline Ischemic disease & $20(33.9)$ & $11(27.8)$ & \\
\hline \multicolumn{4}{|l|}{ Underlying disease $(n, \%)$} \\
\hline Hypertension & $33(55.9)$ & $20(55.6)$ & .97 \\
\hline $\mathrm{DM}$ & $14(24.1)$ & $10(27.8)$ & .69 \\
\hline Heart disease & $9(15.3)$ & $10(27.8)$ & .13 \\
\hline Perioperative use of anticoagulants $(n, \%)$ & $8(13.6)$ & $4(11.1)$ & $>.99$ \\
\hline $\begin{array}{l}\text { BMI }=\text { body mass index } \\
\text { GCS }=\text { Glasgow coma scale } \\
\text { ICH }=\text { intracerebral hemorrhage } \\
\text { SAH = subarachnoid hemorrhage } \\
\text { DM }=\text { diabetes mellitus }\end{array}$ & & & \\
\hline
\end{tabular}

Table 2. Comparison of the Incidence of Complications Between Early and Standard Tracheostomy

\begin{tabular}{|c|c|c|c|}
\hline & \multicolumn{2}{|c|}{ Subjects, $n,(\%)$} & \multirow[b]{2}{*}{$P$} \\
\hline & $\begin{array}{l}\text { Early Tracheostomy } \\
\quad(n=59)\end{array}$ & $\begin{array}{c}\text { Standard Tracheostomy } \\
\quad(n=36)\end{array}$ & \\
\hline Early complications & 5 & 2 & .70 \\
\hline Hemorrhage & $3(5.1)$ & $1(2.8)$ & \\
\hline Subcutaneous emphysema & $1(1.7)$ & $1(2.8)$ & \\
\hline Tube obstruction & $1(1.7)$ & 0 & \\
\hline Tracheal wall injury & 0 & 0 & \\
\hline Late complications & 13 & 3 & .08 \\
\hline Persistent stoma & $6(10.2)$ & $2(5.6)$ & \\
\hline Granuloma formation & $6(10.2)$ & 0 & \\
\hline Tube displacement & 0 & $1(2.8)$ & \\
\hline Wound infection & $1(1.7)$ & 0 & \\
\hline Tracheal stenosis & 0 & 0 & \\
\hline Total complications & $18(30.5)$ & $5(13.9)$ & .06 \\
\hline
\end{tabular}

and 2 cases of subcutaneous emphysema. Two cases of laryngotracheal stenosis and one case of tracheoinomminate fistula were also reported as late complications (occurring after $30 \mathrm{~d}) .{ }^{14}$ Another study investigating adverse events following tracheostomy reported an incidence rate of $39 \%$. In that study, hypoxemia was the most frequent intra-operative adverse event, and stoma inflammation was the most frequent postoperative adverse event. ${ }^{15}$

Although numerous studies in the past few years have reported benefits of early tracheostomy, the specific benefits described in these studies varied. Jeon et al ${ }^{16}$ reported that early tracheostomy in critically ill neurosurgical patients reduced the duration of mechanical ventilation, the length of the stay in the ICU, and the incidence of ventilator-associated pneumonia, but did not reduce ICU and hospital mortality. Bösel et $\mathrm{al}^{7}$ reported that early tracheostomy performed 1-3 d after intubation in stroke patients reduced ICU mortality, use of sedatives, and 6-month mortality. Finally, Devarajan et al ${ }^{8}$ reported that early tracheostomy in patients requiring postoperative mechanical ventilation following cardiac surgery was more effective compared with late tracheostomy in reducing hospital mortality, cardiac mortality, and the duration of ICU and hospital stays.

In contrast to these reports, several studies have reported minimal benefits of early tracheostomy. Young 


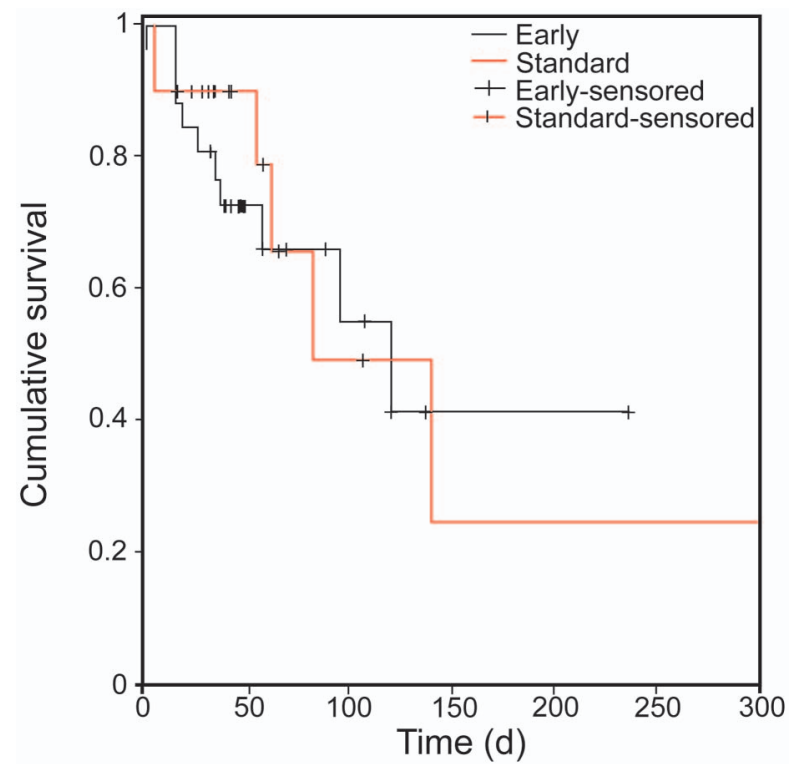

Fig. 2. Kaplan-Meier comparison of the survival time of early and standard tracheostomy subject groups ( $P=.76$, log-rank test).

et a ${ }^{17}$ reported that tracheostomy performed within $4 \mathrm{~d}$ of critical care admission in patients requiring mechanical ventilation was not correlated with improvements in 30-d mortality and other important secondary outcomes. Koch et $\mathrm{al}^{18}$ reported that, although early tracheostomy offered many advantages such as reducing the time of ventilation and hospitalization, it failed to reduce mortality rate in critically ill patients. Finally, Terragni et al ${ }^{15}$ compared the incidence of ventilator-associated pneumonia in mechanically ventilated adult ICU patients and found no significant difference $(P=.07)$ between the early tracheostomy group (14\%) and the late tracheostomy group (21\%).

In our study, we also found no significant difference in the incidence of complications between subjects undergoing early versus standard tracheostomy. Early tracheostomy is known to provide many benefits for patients who require prolonged mechanical ventilation, and is advised if the patient's condition requires this intervention. However, it is necessary to consider the appropriate timing of this procedure by taking into account the possible complications of tracheostomy. A study by Ganuza et a ${ }^{19}$ showed no significant difference in the number of complications between early and late tracheostomy in patients with acute traumatic spinal cord injury, which is consistent with the present study. However, they found that the development of tracheal stenosis was associated with late tracheostomy. Interestingly, in our study, standard tracheostomy had a higher incidence of late complications (granuloma and persistent stoma) than early tracheostomy, although this difference was not statistically significant. Early tracheostomy could result in a reduction in sedative use because tracheostomy is far better tolerated than an endotracheal

Table 3. Risk Factors for Tracheostomy-Related Complications

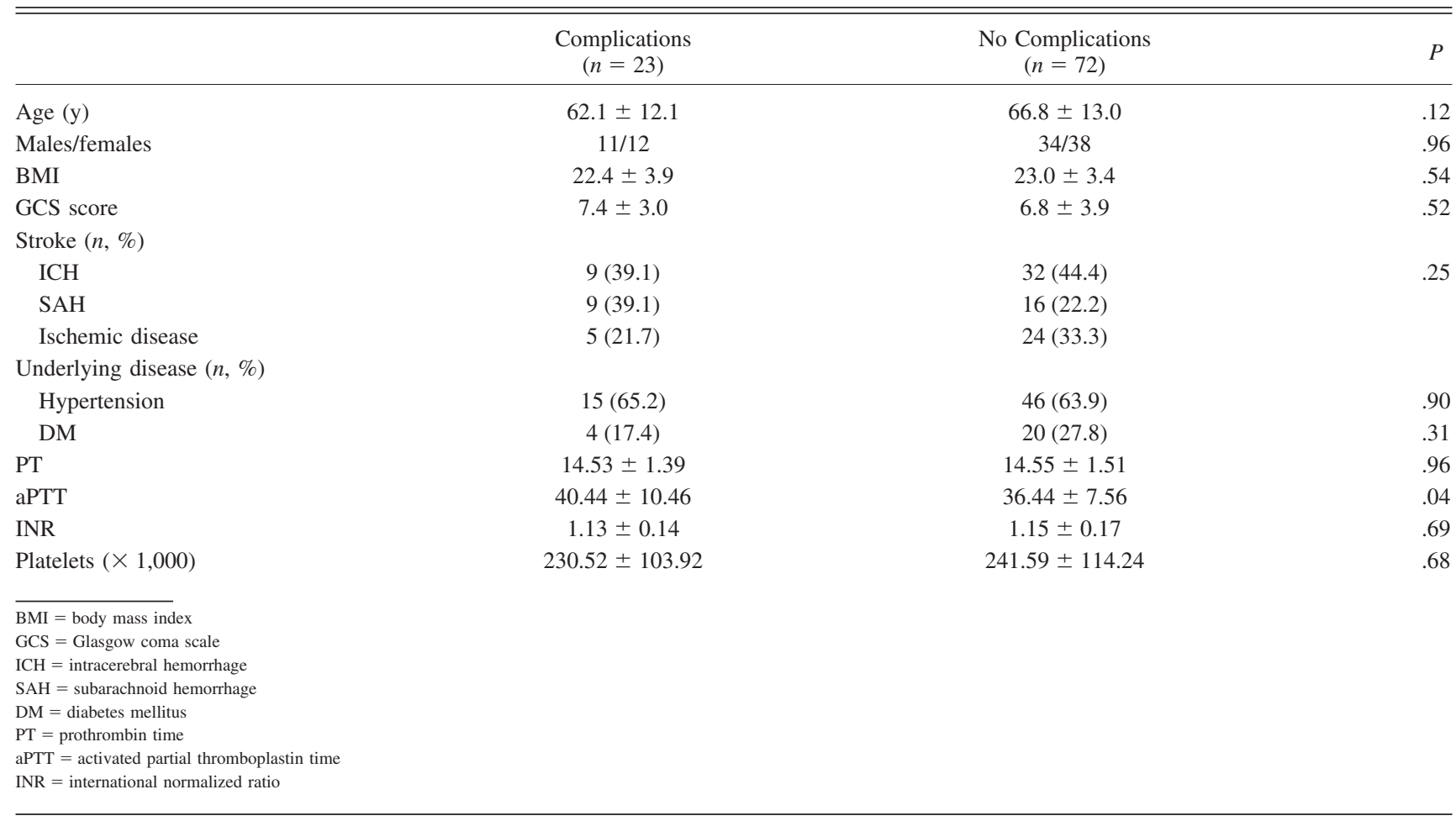




\section{Complications in Early vs Standard Tracheostomy}

tube. ${ }^{17}$ Movement of the tube within the stoma and tracheal lumen in patients while awake may cause ulceration and increase the development of granulation and epithelization of the stoma. ${ }^{20}$ This could be a possible explanation for the increased incidence of granulation and persistent stoma in early tracheostomy.

According to a previous study, risk factors for tracheostomy-related complication include morbid obesity (BMI > 35) and coagulopathy (international normalized ratio $>1.5$, platelet count $<20,000$, or systemic heparinization). ${ }^{21}$ In this study, BMI, prothrombin time, aPTT, international normalized ratio, and platelet count of the groups with and without complications were evaluated, and only aPTT, which was higher in the complications group, was significantly different between patients with and without complications. Therefore, preoperative coagulopathy must be taken into consideration.

Although the morbidity and mortality associated with open surgical tracheostomy have decreased, the use of percutaneous tracheostomy in ICUs is rapidly increasing because of its lower complexity. ${ }^{22-24}$ The minimal dissection involved in percutaneous tracheostomy results in less tissue damage, lowers the incidence of bleeding and wound infection, and can be safely performed at the bedside in the ICU. ${ }^{24,25}$ Kost $^{26}$ reported that the overall complication rate for 500 cases of endoscopic percutaneous dilatational tracheostomy was $9.2 \%$, with two-thirds of these considered minor in nature. The present study did not include any cases of percutaneous dilatational tracheostomy. Therefore, further investigation of the influence of timing on the incidence of complication associated with percutaneous tracheostomy is necessary.

One limitation of this study is that identification of complications relied on retrospective chart review, and, thus, some complications may have been omitted during this process. Furthermore, we did not utilize a quantifiable assessment of disease in stroke patients such as Acute Physiology and Chronic Health Evaluation (APACHE) or Sequential Organ Failure Assessment (SOFA). Finally, although each tracheostomy was performed by a skilled otolaryngologist or neurosurgeon, the fact that not all tracheostomies were performed by the same surgeon may have affected the incidence of complications because of slight variations in technique. However, the main steps of the procedure were nearly identical for all tracheostomies, and the lack of unusual events during tracheostomy in any of the subjects included in this study suggests that these effects were probably negligible.

\section{Conclusions}

There was no significant difference in the incidence of complications between stroke subjects undergoing early versus standard tracheotomy.

\section{REFERENCES}

1. Armstrong PA, McCarthy MC, Peoples JB. Reduced use of resources by early tracheostomy in ventilator-dependent patients with blunt trauma. Surgery 1998;124(4):763-766; discussion 766-767.

2. Arabi Y, Haddad S, Shirawi N, Al Shimemeri A. Early tracheostomy in intensive care trauma patients improves resource utilization: a cohort study and literature review. Crit Care 2004;8(5):R347-R352.

3. Kanna B, Ayman HA, Soni A. Early tracheostomy in intensive care trauma patient improves resource utilization: a cohort study and literature review. Crit Care 2005;9(4):414-416;author reply 414-416; discussion 414-416.

4. Schneider GT, Christensen N, Doerr TD. Early tracheotomy in elderly patients results in less ventilator-associated pneumonia. Otolaryngol Head Neck Surg 2009;140(2):250-255.

5. Rumbak MJ, Newton M, Truncale T, Schwartz SW, Adams JW, Hazard PB. A prospective, randomized, study comparing early percutaneous dilational tracheotomy to prolonged translaryngeal intubation (delayed tracheotomy) in critically ill medical patients. Crit Care Med 2004;32(8):1689-1694.

6. Yavas S, Yagar S, Mavioglu L, Cetin E, Iscan HZ, Ulus AT, Birincioglu CL. Tracheostomy: how and when should it be done in cardiovascular surgery ICU? J Card Surg 2009;24(1):11-18.

7. Bösel J, Schiller P, Hook Y, Andes M, Neumann JO, Poli S, et al. Stroke-related Early Tracheostomy versus Prolonged Orotracheal Intubation in Neurocritical Care Trial (SETPOINT): a randomized pilot trial. Stroke 2013;44(1):21-28.

8. Devarajan J, Vydyanathan A, Xu M, Murthy SM, McCurry KR, Sessler DI, et al. Early tracheostomy is associated with improved outcomes in patients who require prolonged mechanical ventilation after cardiac surgery. J Am Coll Surg 2012;214(6):1008-1016 e1004.

9. De Leyn P, Bedert L, Delcroix M, Depuydt P, Lauwers G, Sokolov Y, et al. Tracheotomy: clinical review and guidelines. Eur J Cardiothorac Surg 2007;32(3):412-421.

10. Heffner JE, Miller KS, Sahn SA. Tracheostomy in the intensive care unit. Part 1: Indications, technique, management. Chest 1986;90(2): 269-274.

11. Freeman BD, Borecki IB, Coopersmith CM, Buchman TG. Relationship between tracheostomy timing and duration of mechanical ventilation in critically ill patients. Crit Care Med 2005;33(11):25132520.

12. Frutos-Vivar F, Esteban A, Apezteguía C, Anzueto A, Nightingale P, González M, et al. Outcome of mechanically ventilated patients who require a tracheostomy. Crit Care Med 2005;33(2):290-298.

13. Nathens AB, Rivara FP, Mack CD, Rubenfeld GD, Wang J, Jurkovich GJ, Maier RV. Variations in rates of tracheostomy in the critically ill trauma patient. Crit Care Med 2006;34(12):2919-2924.

14. Terra RM, Fernandez A, Bammann RH, Castro AC, Ishy A, Junqueira JJ. Open bedside tracheostomy: routine procedure for patients under prolonged mechanical ventilation. Clinics (Sao Paulo) 2007; 62(4):427-432.

15. Terragni PP, Antonelli M, Fumagalli R, Faggiano C, Berardino M, Pallavicini FB, et al. Early vs late tracheotomy for prevention of pneumonia in mechanically ventilated adult ICU patients: a randomized controlled trial. JAMA 2010;303(15):1483-1489.

16. Jeon YT, Hwang JW, Lim YJ, Lee SY, Woo KI, Park HP. Effect of tracheostomy timing on clinical outcome in neurosurgical patients: early versus late tracheostomy. J Neurosurg Anesthesiol 2014;26(1):22-26.

17. Young D, Harrison DA, Cuthbertson BH, Rowan K. Effect of early vs late tracheostomy placement on survival in patients receiving mechanical ventilation: the TracMan randomized trial. JAMA 2013; 309(20):2121-2129. 


\section{Complications in Early vs Standard Tracheostomy}

18. Koch T, Hecker B, Hecker A, Brenck F, Preuss M, Schmelzer T, et al. Early tracheostomy decreases ventilation time but has no impact on mortality of intensive care patients: a randomized study. Langenbecks Arch Surg 2012;397(6):1001-1008.

19. Ganuza JR, Garcia Forcada A, Gambarrutta C, Diez De La Lastra Buigues E, Merlo Gonzalez VE, Paz Fuentes F, Luciani AA. Effect of technique and timing of tracheostomy in patients with acute traumatic spinal cord injury undergoing mechanical ventilation. J Spinal Cord Med 2011;34(1):76-84.

20. Yaremchuk K. Regular tracheostomy tube changes to prevent formation of granulation tissue. Laryngoscope 2003;113(1):1-10.

21. Blankenship DR, Kulbersh BD, Gourin CG, Blanchard AR, Terris DJ. High-risk tracheostomy: exploring the limits of the percutaneous tracheostomy. Laryngoscope 2005;115(6):987-989.
22. Myers EN, Carrau RL. Early complications of tracheotomy. Incidence and management. Clin Chest Med 1991;12(3):589-595.

23. Waldron J, Padgham ND, Hurley SE. Complications of emergency and elective tracheostomy: a retrospective study of 150 consecutive cases. Ann R Coll Surg Engl 1990;72(4):218-220.

24. Al-Ansari MA, Hijazi MH. Clinical review: percutaneous dilatational tracheostomy. Crit Care 2006;10(1):202.

25. Delaney A, Bagshaw SM, Nalos M. Percutaneous dilatational tracheostomy versus surgical tracheostomy in critically ill patients: a systematic review and meta-analysis. Crit Care 2006; 10(2):R55.

26. Kost KM. Endoscopic percutaneous dilatational tracheotomy: a prospective evaluation of 500 consecutive cases. Laryngoscope 2005; 115(10 Pt 2):1-30.

This article is approved for Continuing Respiratory Care Education credit. For information and to obtain your CRCE

(free to AARC members) visit 